\title{
Blood Generation from Stem Cells: An Overview
}

\author{
Vimal Kishor Singh $^{1 *}$, Abhishek Saini ${ }^{1}$, Neeraj Kumar ${ }^{1}$, Manisha Kalsan ${ }^{1}$ \\ ${ }^{1}$ Stem Cell Research Laboratory, Department of Biotechnology, Delhi Technological University, Delhi \\ ${ }^{2}$ Correspondence: Dr. Vimal Kishor Singh O/I Stem Cell Research Laboratory, Department of Biotechnology, \\ Delhi Technological University, Delhi, India
}

\begin{abstract}
Blood transfusions are the mainstay in modern clinical modalities. The worldwide spread blood banks secure the supply of various blood components which solely depend upon voluntary paid/unpaid donor. According to the WHO reports present collections and supply of safe/adequate blood is inadequately distributed among economically developed and developing countries. Further, blood transfusions are prone to a number of risks/ adverse reactions that may be imposed due to inefficient blood screening and processing methods and put extreme supply constraints, especially in the underdeveloped countries. In past few years stem cells from different sources are demonstrated to give rise to the significant amount of functionally mature erythrocytes, platelets and neutrophils by using a cocktail of growth factors under standard culture conditions. Therefore, a logistic approach to ex vivo manufacturing of erythrocytes (\& other blood components) through modern tissue culture techniques has surfaced in the past few years. Ex vivo expansion of erythrocytes to generate transfusable units of clinical grade RBCs from a single dose of starting material (e.g. umbilical cord blood) has been well established. More and more information is coming from the various research groups showing the use of other potential sources (e.g. embryonic stem cells, induced pluripotent stem cells, and stress induced erythroid progenitors), different cocktails of growth factors, methodologies to get optimal yields of the functional RBCs/ blood cells. One can imagine that with the advent of more defined methods for large-scale production of blood cells the existing system of donor derived blood supplies could be replaced in future. However, various regulating factors should essentially control the fate of large-scale production of RBCs including issues related to the quantity/ quality and per unit cost of these products. These issues would be solved with the invention of more automated culture techniques such as the use of bioreactors and identification of more direct molecular agents regulating all phases of their ex vivo generation such as stem cell proliferation, differentiation, and maturation of final products. Recent studies have shown the possibilities of generating several hundred units of erythrocytes by using continuously feeding hollow fiber bioreactors along with other types of bioreactors. Even more, the functionality and acceptability of ex vivo cultured RBCs is demonstrated by their in-human transfusion which provides essential motivation to develop more sophisticated methods/culturing techniques to pave the way for commercial production of blood components at a feasible cost.
\end{abstract}

Keywords: Ex vivo RBCs expansion, Blood Pharming, IPSCs, RBCs, Manufacturing blood, CD34 ${ }^{+}$HSCs.

\section{Introduction}

Modern clinical modalities revolve around blood bank system which is an inevitable part of almost all the surgical interventions and support all kinds of blood transfusion needs [1]. According to the WHO reports about103 million blood units are collected across the globe every year [2]. However, huge discrepancies occur among economically developed and developing countries as their accessibility to safe and adequate blood supplies are concerned. The people living in developing countries have limited supplies of safe blood as compared to the economical developed countries. Developed countries have a small population (15\% of the global population) but have access to more than $50 \%$ of the total global blood collection/supplies per annum. WHO reports states a higher rate of blood donations scored (>30,000 annual blood donations/per blood center) by 8000 blood centers scattered in 159 high-income countries [3]. This indicates that safe and sufficient blood units are comparatively easily available in the developed countries and the blood collection seems to be sufficient for the time being at least. While, the situation is not so comfortable in economically developing countries where only 3700 blood collections per center are reported. Further, almost $80 \%$ of the world population resides in these countries which reduces the per person availability of the adequate blood supplies up to a significant level. This is reported by WHO showing that there are only 10 donations per 1000 people in the 82 low income and middle income countries [4]. The situation is worsened due to inefficient screening facilities available in most of the underdeveloped countries. It is reported that $\sim 39$ countries lack efficient screening facilities for most severe transfusion-transmissible infections (TTIs) viz. HIV, hepatitis B, hepatitis C, and syphilis [4]. Further, only 106 countries are equipped with national blood transfusion guidelines and only $13 \%$ of the low-income countries have established a national haemovigilance system to monitor the quality of the transfusion processes. An important fact is that the proportion of elderly population is rapidly growing most developed countries ( $>60$ years old) and in the absence of young donors it would be difficult to meet the demands for blood transfusions even in the developed countries by the year of 2050 [5].

In the absence of sufficient blood inventories, it would be hard to find exact match for $>30$ blood group system with 308 recognized antigens including $\mathrm{ABO} \& \mathrm{Rh}$ antigens [6]. In addition, there are demands for rare phenotypic blood, patients with various hemoglobinopathies, polytransfusion patients and polyimmunization which put more constraints on the present donor derived blood supply system across the globe [4]. Although, there are few reports showing procedures to develop universal blood group by using antigen masking and/or enzymatic cleavage techniques but these methods are not yet developed properly [7]. Apart from these supplies related problems, the association of various types of adverse reactions with the donor derived blood 


\section{International Journal of Science and Research (IJSR) \\ ISSN (Online): 2319-7064 \\ Index Copernicus Value (2013): 6.14 | Impact Factor (2014): 5.611}

supplies system has been challenging the clinicians to find more suitable alternatives to avoid these problems. Although, a number of testing, preclinical precautionary measures are taken to avoid any infectious agent [e.g., HIV-1, HIV-2, HTLV-1, HTLV-2, Hepatitis B, Hepatitis C, Syphilis (T pallidum), Chagas disease ( $T$ cruzi), and West Nile Virus, Cytomegalovirus (CMV)], but blood transfusions are often subjected to cause several complicated situations [1, 3-4]. These adverse reactions/complications are reported to causes delayed cure rate with prolonged hospitalization periods and may further raise the overall cost of treatment [3]. For the last few years, researchers have been trying to find various non-donor derived sources such as artificial blood. However, the majority of artificial blood molecules such as hemoglobin-based oxygen carriers or perfluorocarbon solutions remains inefficient oxygen carriers and thus have limited functional application [8-12].

Another more promising approaches are the ex vivo expansion of erythrocytes from stem cells. As discussed in the later sections of the article a number of protocols have been derived showing a significant amount of functionally matured RBCs by using different types of stem cells.

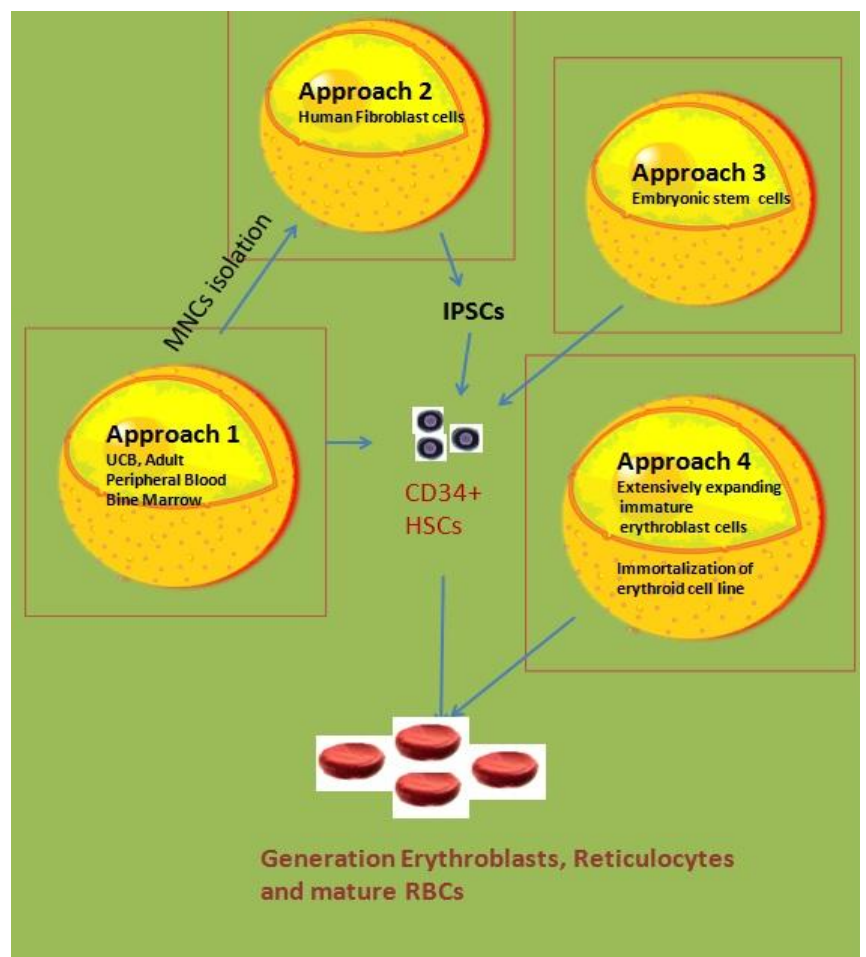

Figure 1: Various approaches in use for Ex vivo RBCs expansion: Ex vivo generation of transfusable units of RBCs can be done through different source materials and methodologies as shown above. CD34+ HSPCs (may be isolated from $C B, P B$, and $B M$ ), ESCs/IPSCs derived erythroid progenitors, and highly expanding erythroid progenitors due to stress erythropoiesis are equally potent but the overall yield, degree of maturation, methodologies used are differently developed by various research groups Ex

Vivo Erythropoiesis and Different Approaches

The first report came from Nakamura and his colleague who demonstrated survival of lethally irradiated mice through transfusion of erythroid progenitors/ cells which was generated by ex vivo differentiation of Embryonic Stem cell line (ESCs) [13].

These findings tempted many researchers who eventually developed various protocols to derive functionally matured erythrocytes from different types of stem cells viz. CD34+ HSCs, Embryonic stem cells, and IPSCs based methods (Figure 1). The clinical utility of cultured RBCs was established through proof-of-concept study by Giarratana et al, who shown first in-human blood transfusion of ex-vivo generated RBCs [14]. These studies indicated that expansion of erythrocytes from stem cells could become a powerful tool to solve the problems of blood supply shortages and other associated issues. With the advent of more suitable efficient culturing protocols a regular mass scale production could be feasible in near future. However, a number of relevant issues need to be defined to ensure the sufficient yields of transfusable RBCs units through these protocols. Fortunately, people have demonstrated possibilities of numerical expansion of erythrocyte/ precursors by using similar techniques. Umbilical cord blood-derived CD34+ cells can be expanded $\sim 2$ million fold to give rise to enucleated erythroid cells that are equivalent to $\sim 0.5$ transfusion units of donor derived blood [15]. Similarly, UCB derived CD34+ are reported to generate 10 transfusion unit equivalents of RBCs by a technically complex protocol [16]. Although, these primitive methods are not best suitable for the large scale commercial production, but their potential up gradation to scale-up the overall production would become feasible in near future. Another lacuna is the sub-optimal enucleation efficiency of RBCs population generated by these protocols indicating their incomplete functional maturation. However, it is believed that these nucleated RBCs precursors, cellular intermediates/nucleated erythroid precursors generated through ex vivo expansion methods may also be of use if transfused. It is proposed that these maturing nucleated cells along with enucleated erythrocytes can support the cellular content at the time of transfusion and may also give rise to functionally matured cell by proliferating into 4-64 enucleated RBCs in circulation. This is supported by reports showing functional maturation of cellular intermediates/nucleated erythroid precursors in vivo on posttransfusion through their interaction with internal factors regulating their maturation. There are further studies both in human and animal models showing similar reports [15, 17]. This is also supported by several clinical reports showing occasional use of compatible Cord Blood (40-80 ml/4-7 $\times 10^{10} \mathrm{RBCs}$ and $2-8 \times 10^{9}$ erythroblast cells) for transfusion in emergency situations [17].

These reports support the feasibilities of regular ex vivo RBCs expansion to overcome different clinical short-comes that exist because of supply constraints. However existing technological barriers need development of more automated and efficient ex vivo expansion methods for large scale production. In order to enhance the present scale of RBCs production a more cost-effective and timely automated ex vivo culture approaches are required. This is possible if we 


\section{International Journal of Science and Research (IJSR) \\ ISSN (Online): 2319-7064}

Index Copernicus Value (2013): 6.14 | Impact Factor (2014): 5.611

have an in-depth knowledge of the various regulatory elements and their mode of action playing axial role in almost all of these methods demonstrated so far.

The present article focuses on the various approaches developed for ex vivo erythrocyte expansion and other important regulatory elements to discuss the fate of largescale RBCs production.

During past few decades considerable progress has occurred in the understanding and therefore developing methods for biological control over the expansion of erythrocytes from hematopoietic stem cells. This information is very well exploited to generate terminally differentiated, fully functional RBCs [18]. There are different types of stem cells such as CD34+ HSPCs, Embryonic stem cells/induced pluripotent stem cells, and immortalized erythroid precursors which can be differentiated into erythroid lineages by using almost similar/overlapping protocols (Figure2). The overall ex vivo expansion process may be categorized into three broad phases Such as phase I: -commitment, phase II: expansion, and phase III: maturation or enucleation. Recent studies has demonstrated transdifferentiation (directly differentiating of human fibroblast cells into erythroblasts) to bypass the HSPC state (Figure3) [19]. These reports are promising and a detailed technical evaluation of their potential to generate large-scale RBCs would be helpful in establishing the essential guidelines.

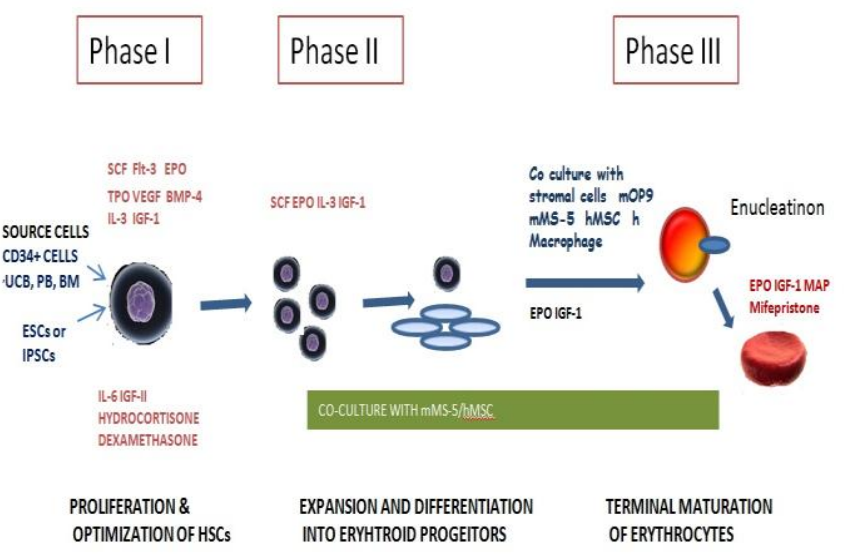

Figure 2: An overview of the methodologies/protocols: Ex vivo RBCs expansion/ manufacturing blood may involve various phases such as depicted above. Phase 1deals with the initial source material collection from a variety of source material(s).these cells grown are on the basis of their availability, suitability, and expansion potential in medium generally supplemented with growth factors to enhance HSCs proliferation: subsequently, phase II entails culture of these cells in the presence of Erythropoietin to induce their differentiation and maintenance into erythropoietic progenitor stage. The final Phase has been described to support their maturation. Here, Erythropoietic progenitors may be co cultured with murine/human stromal cell line support to induce their maturation and enucleation resulting into mature RBCs. These RBCs are evaluated for their biochemical properties and various antigenic profiles to ensure their nativeness.

\section{The Fate of Blood Production is Regulated by Various Factors}

Efforts are being made develop optimal production methods by controlling different variables of ex vivo RBCs expansion procedures. There are a number of important factors which may be summarized as follow:

1) Issues related to the initial source material

2) Quantitative issues

3) Growth factors and their optimal uses

4) Biophysical and biochemical parameters

5) Issues related to the antigenic profiling

6) Ethnicity-related issues

7) Issues related to the enucleation efficiency of cultured RBCs

8) Production cost

The most important factor is the initial source material for various methods that are being explored for large scale RBCs production. An ideal source material would be a discarded material (reducing production cost), available in abundance, possess high potential to give rise to final product i.e. matured RBCs. and non-immunogenic. Most of these criteria fits well with umbilical $\mathrm{CB}$ which is easily available and have been used to produce sufficient amount of transfusable RBCs.

Another important factor is the extremely huge quantity of any blood component that would be required to produce. For example, RBCs are present in $2 \times 10^{12}$ cells/unit $(200 \mathrm{ml}$ of blood) with an expected cell density of $3 \times 106$ cells $/ \mathrm{ml}$. In order to support global population a mammoth amount of blood units (e.g. >103 million units) would be an essential requirement. Considering the present ex vivo expansion technologies an extremely large amount of ingredients and adequately skilled manpower would be an inevitable requirement. This is hypothesized that a cell density of $5 \times 10^{7}$ cells/ml should be produced regularly to support this much demand. It is estimated that production of single unit of blood through present static culture methods will require 660 liters of culturing medium and 9500 lab scale $175 \mathrm{~cm}^{2}$ culturing flasks (70ml medium/flask) [20-22]. This indicates the need for more automated 3D culture techniques to ensure such high production efficiency.

Fortunately, use of bioreactors may be helpful in reducing both the culturing assets and associated labor. This could help researchers achieve required cell density with limited efforts. It is reflected from the reports showing production of large density of RBCs in a wave bioreactor [22].

Similar results are reported (cell density of $10^{7}$ cells/ml) by using a small-scale stirred bioreactor [23]. Further, a massive yield of $2 \times 108$ cells $/ \mathrm{ml}$ was produced in a hollow fiber bioreactor [24]. The point is that with the advent of the technology these 3D bioreactors may be scaled up with to produce desired units of RBCs in 3-4 weeks of time. Thus it seems feasible to produce large amounts of RBCs, but before that many factors such as degree of cell maturation and 


\section{International Journal of Science and Research (IJSR) \\ ISSN (Online): 2319-7064 \\ Index Copernicus Value (2013): 6.14 | Impact Factor (2014): 5.611}

various cultural parameters should be defined in detail. Next, there are few reports showing the preliminary data on direct transformation of stem cells into the erythropoietic lineages which will dramatically reduce both the time and cost of production. For example, generation of erythropoietic cell lines from ESCs/IPSCs through reprogramming of any somatic cells directly into erythroblasts using suitable genes is reported by some researchers. Cheng's group has reported in humans that the differentiated erythroid cells obtained from human embryo might be used as a potential initial source for RBCs production [25-26].

In brief, it is conceivable that limited production of RBCs may take place in near future. The upcoming advancements in the existing tissue/ cell culture technology and identification of most efficient source materials would ensure sufficient ex vivo expansion of RBCs/ other blood cells in a regular fashion. The quality issues of these products may be resolved with the development of highly stringent, comprehensive quality control, analytical measures. As discussed earlier, all types of stem cells (e.g. CD34+ HSCs, hESCs and, iPSCs) have enough potential to support the production of clinical grade blood cells in huge quantities and invention of more efficient source materials like erythropoietic hESC cell lines which can be directly transformed/induced to generate erythrocytes, would further ensure the unlimited supply these products. Since a number of growth factors are used in these protocols and the molecular mechanisms involved in various stages of ex vivo erythropoiesis governed through these growth factors is not precisely defined. The exploration of these fundamental processes would be helpful in developing pharmacological alternates for the various stages of erythropoietic development.

Next, use of new pharmacological alternates can further simplify the production processes for clinical grade blood by removing the need for feeder cells and thus may help in reducing the unexpected risks associated with their use due to undefined immunogenic and infectious exposures. The omission of feeder cells from these methods is not only essential for the generation of GMP products but is also required to simplify the commercial scale production methods. Use of straightforward and simple procedures may also be helpful in reducing the cost of production.

\section{Acknowledgement}

We are thankful to the Honorable Chairman and the Honorable Vice-Chancellor of the Delhi Technological University, Shahbad Daulatpur, Bawana road, Delhi-42, for support. Dr. Vimal K. Singh particularly thanks the Department of Science and Technology and Indian National Science Academy (INSA), INDIA, for the research grant.

\section{Conflict of Interest}

The authors certify that they have NO affi liations with or involvement in any organization or entity with any financial interest (such as honoraria; educational grants; participation in speakers' bureaus; membership, employment, consultancies, stock ownership, or other equity interest; and expert testimony or patent-licensing arrangements), or nonfinancial interest (such as personal or professional relationships, affiliations, knowledge or beliefs) in the subject matter or materials discussed in this manuscript.

\section{References}

[1] Alter, H. J., and Klein, H. G. (2008). The hazards of blood transfusion in historical perspective. Blood 112, 2617-2626. doi: 10.1182/blood-2008-07-077370

[2] World Health Organization. (2011). Global Database on Blood Safety: Summary Report 2011. Available online at: http://www.who.int/bloodsafety/ global_database/GDBS_Summary_Report_2011.pdf (Accessed May 4, 2014).

[3] Department of Health and Human Services. (2010). The 2009 National Blood Collection and Utilization Survey Report. Washington, DC: DHHS. Available online at: http://www.aabb.org/programs/biovigilance/nbcus/Docu ments/ 09-nbcus-report.pdf (Accessed May 4, 2014).

[4] Department of Health and Human Services. (2013). The 2011 National Blood Collection and Utilization Survey Report. Washington, DC: DHHS. Available online at: http://www.aabb.org/programs/biovigilance/nbcus/Docu ments/ 11-nbcus-report.pdf (Accessed May 4, 2014).

[5] U.S. Census Bureau. (2004). Global Population Composition. Available online at:http://www.census.gov/population/international/files/ wp02/wp-02004.pdf (Accessed September 14, 2012).

[6] Daniels, G., Castilho, L., Flegel, W. A., Fletcher, A., Garratty, G., Levene, C., et al. (2009). International society of blood transfusion committee on terminology for red blood cell surface antigens: macao report. Vox. Sang. 96, 153-156. doi: 10.1111/j.14230410.2008.01133.x

[7] Bagnis, C., Chiaroni, J., and Bailly, P. (2011). Elimination of blood group antigens: Hope and reality.Br. J. Haematol. 152, 392-400. doi: 10.1111/j.13652141.2010.08561.x

[8] Winslow, R. M. (2006). Current status of oxygen carriers ("blood substitutes"): 2006. Vox Sang. 91, 102110. doi: 10.1111/j.1423-0410.2006.00789.x

[9] Henkel-Honke, T., and Oleck, M. (2007). Artificial oxygen carriers: a current review. AANA. J. 75, 205211.

[10] Natanson, C., Kern, S. J., Lurie, P., Banks, S. M., and Wolfe, S. M. (2008).Cellfree hemoglobin-based blood substitutes and risk of myocardial infarction and death: a meta-analysis. J. Am. Med. Assoc. 299, 2304-2312. doi: 10.1001/jama.299.19.jrv80007.

[11] Silverman, T. A., and Weiskopf, R. B. (2009). Hemoglobinbased oxygen carriers: Current status and future directions. Transfusion 49, 2495-2515. doi: 10.1111/j.1537-2995.2009.02356.x.

[12] Castro, C. I., and Briceno, J. C. (2010). Perfluorocarbonbased oxygen carriers: Review of products and trials. Artif. Organs 34, 622-634. doi: 10.1111/j.15251594.2009.00944.X

[13] Hiroyama, T., Miharada, K., Sudo, K., Danjo, I., Aoki, N., and Nakamura, Y. 


\section{International Journal of Science and Research (IJSR) \\ ISSN (Online): 2319-7064 \\ Index Copernicus Value (2013): 6.14 | Impact Factor (2014): 5.611}

(2008).Establishmentofmouseembryonicstemcellderivederythroidprogenitor cell lines able to produce functional red blood cells. PLoS ONE 6:3. doi: 10.1371/journal.pone.0001544.

[14] Giarratana, M. C., Rouard, H., Dumont, A., Kiger, L., Safeukui, I., Le Pennec, P. Y., et al. (2011). Proof of principle for transfusion of in vitro-generated red blood cells. Blood 118, 5071-5079. doi: 10.1182/blood-201106-362038

[15] NeildezNguyen,T.M.,Wajcman,H.,Marden,M.C.,Bensid houm,M.,Moncollin, V., Giarratana, M. C., et al. (2002). Human erythroid cells produced ex vivo at large scale differentiate in to red blood cells in vivo.Nat.Biotechnol.20,467-472. doi: 10.1038/nbt0502467.

[16] Fujimi, A., Matsunaga, T., Kobune, M., Kawano, Y., Nagaya, T., Tanaka, I., et al. (2008).Ex vivo large-scale generation of human red blood cells from cordblood CD34+ cells by co-culturing with macrophages. Int. J. Hematol. 87, 339-350. doi: 10.1007/s12185-008-0062-y

[17] Ende, M., and Ende, N. (1972). Hematopoietic transplantation by means of fetal (cord) blood. A new method. Va. Med. Mon. 99, 276-280.

[18] Migliaccio, A. R., Masselli, E., Varricchio, L., and Whitsett, C. (2012). Ex-vivo expansion of red blood cells: how real for transfusion in humans? Blood Rev. 26, 81-95. doi: 10.1016/j.blre.2011.11.002

[19] Szabo, E., Rampalli, S., Risueno, R. M., Schnerch, A., Mitchell, R., Fiebig-Comyn, A., et al. (2010). Direct conversion of human fibroblasts to multilineage blood progenitors. Nature 468, 521-526. doi: 10.1038/nature09591

[20] Timmins, N. E., and Nielsen, L. K.,(2009). Blood cell manufacture: current methods and future challenges. Trends Biotechnol. 27, 415-422. doi: 10.1016/j.tibtech.2009.03.008

[21]Zeuner, A., Martelli, F., Vaglio, S., Federici, G., Whitsett, C., and Migliaccio, A. R. (2012). Concise review: stem cell-derived erythrocytes as upcoming players in blood trans- fusion. Stem Cells 30, 15871596. doi: 10.1002/stem.1136

[22] Timmins, N. E., Athanasas, S., Günther, M., Buntine, P., and Nielsen, L. K. (2011). Ultrahigh-yield manufacture of red blood cells from hematopoietic stem cells. Tissue Eng. Part. C Methods 17, 1131-1137. doi: 10.1089/ten.tec.2011.0207

[23] Ratcliffe, E1., Glen, K. E., Workman, V. L., Stacey, A. J., and Thomas, R. J. (2012). A novel automated bioreactor for scalable process optimisation of haematopoietic stem cell culture. J. Biotechnol. 161, 387-390. doi: 10.1016/j.jbiotec.2012. 06.025.

[24]Housler, G. J., Miki, T., Schmelzer, E., Pekor, C., Zhang, X., Kang, L., et al. (2012). Compart- mental hollow fiber capillary membrane-based bioreactor technology for in vitro studies on red blood cell lineage direction of hematopoietic stem cells. Tissue Eng. Part. C Methods 18, 133-142. doi: 10.1089/ten.tec. 2011.0305

[25] Huang, X., Shah, S., Wang, J., Ye, Z., Dowey, S. N., Tsang, K. M., et al. (2013). Extensive ex vivo expansion of functional human erythroid precursors established from umbilical cord blood cells by defined factors. Mol. Ther. 22, 451-463. doi: 10.1038/mt.2013.201
[26] Kurita, R., Suda, N., Sudo, K., Miharada, K., Hiroyama, T., Miyoshi, H., et al. (2013). Establishment of immortalized human erythroid progenitor cell lines able to produce enucleated red blood cells. PLoS ONE 8:3 doi: 10.1371/journal.pone.0059890

\section{Author Profile}

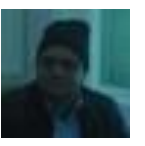

Dr. Vimal Kishor Singh received the M.Sc. in BioMedical Sciences from University of Delhi. PhD from Institute of Nuclear Medicine and Allied Sciences (DRDO)/University of Delhi Founder and O/I of Stem Cell Research Laboratory, Dept. of Biotechnology, Delhi Technological University

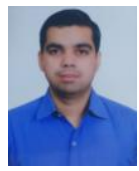

Abhishek Saini received the B.Sc. Microbiology (H) degree from Delhi University and M.Sc. in Biotechnology from TERI University. At present pursuing Ph.D. from Delhi Technological University.

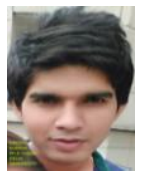

Neeraj Kumar received the B.Sc. degree in Chemistry from Delhi University and M.Sc. degree in Biochemistry from Jamia Millia Islamia University and M.Tech degree in Bioinformatics from Delhi Technological University. Presently, pursuing his Ph.D. from Delhi University.

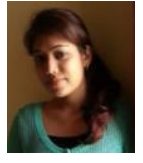

Manisha Kalsan received a B. Tech degree in Biotechnology from D. C. R. University of Science and Technology, Murthal and an $\mathrm{M}$. Tech degree in Bioinformatics from Delhi Technological University and is presently pursuing her Ph.D. from Delhi University. 